\title{
Planets in Binary Systems
}

\author{
Antonio Pilello \\ Institut für Astrophysik, Georg-August-Universität Göttingen, \\ Friedrich-Hund-Platz 1, D-37077 Göttingen, Germany \\ E-Mail: pilello@astro.physik.uni-goettingen.de
}

\begin{abstract}
In close eclipsing binary systems, measurements of the eclipse timing variations (ETV), obtained by means of accurate light curves, may be used to find circumbinary additional objects. The presence of these objects causes the motion of the eclipsing binary with respect to the centre of mass of the entire system and it results in advances or delays in the times of eclipses due to the light time effect. The most important issue of this project is to inspect the potential of detecting low mass substellar companions to close eclipsing binaries through the timing method. For this purpose, we use the public data from Kepler and CoRoT spacecrafts, collecting the light curves for a selected sample and analyzing the observed minus calculated (O-C) times of the eclipses in the search for ETVs and characterizing them. A large amplitude of the O-C ETVs can be explained in some cases by the presence of a third body in the system.
\end{abstract}

Keywords. binaries: eclipsing, methods: data analysis

\section{Introduction}

Kepler and CoRoT light curves are mainly analyzed in the search for planetary transits, but many of eclipsing binary stars (EBs) are also discovered. Sometimes EBs show timing variations $(\mathrm{TVs})$, that are changes in the period of their eclipses. Timing variations of eclipses can provide a comprehension of the physical structure of the system.

\section{Data Analysis}

We use the public data from Kepler and CoRoT space missions, collecting the light curves for a selected sample and analyzing the observed minus calculated $(\mathrm{O}-\mathrm{C})$ times of the eclipses in the search for ETVs and characterizing them. The total number of identified eclipsing binaries in the Kepler field of view is 2165, 1.4\% of the Kepler target stars (Prša et al. 2011, and Slawson et al. 2011, after the Q0, Q1 and Q2 data releases). The EBs with a probability of variability more than $80 \%$ in the N2 CoRoT public data are instead 1707. Several IDL routines are applied to remove the thermal peaks and to flatten the Kepler light curves (four examples are shown in Fig. 1).

The large amplitudes of the O-C eclipse time variations seen in some close binary systems can be explained by the presence of a third body in the system. We present here an interesting case of $\mathrm{EB}$ from Kepler public data with $\mathrm{O}-\mathrm{C}$ variations evident in the Q0, Q1 and Q2 data. In Fig. 2 the normalized light curve and the $\mathrm{O}-\mathrm{C}$ diagram of KID6543674 with a period of 2.390105 days (Slawson et al. 2011) is shown. This object is a short period EB with deep eclipses from two nearly equal components seen close to edge-on. This system has eclipse timing variations, that may arise from the light time effect as the third-body orbits that binary (Slawson et al. 2011). 

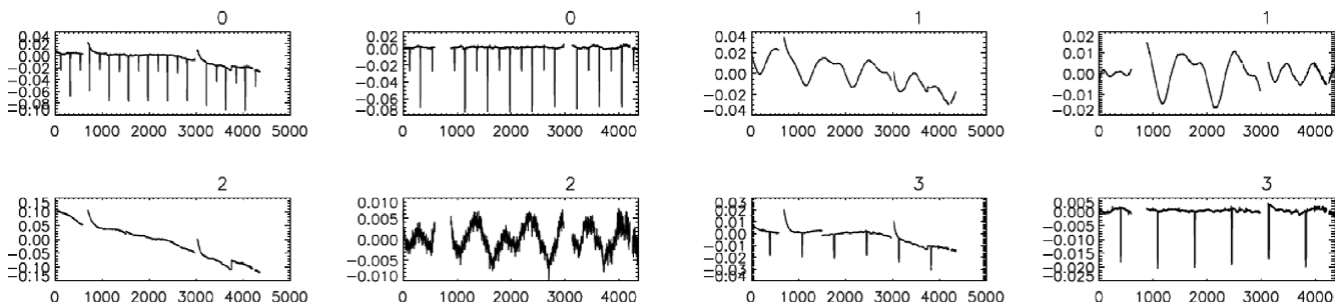

Figure 1. Flatted light curves without thermal effects. The plots with the same index represent the normalized (on the left) and the corrected (on the right) light curve for the corresponding Kepler object.
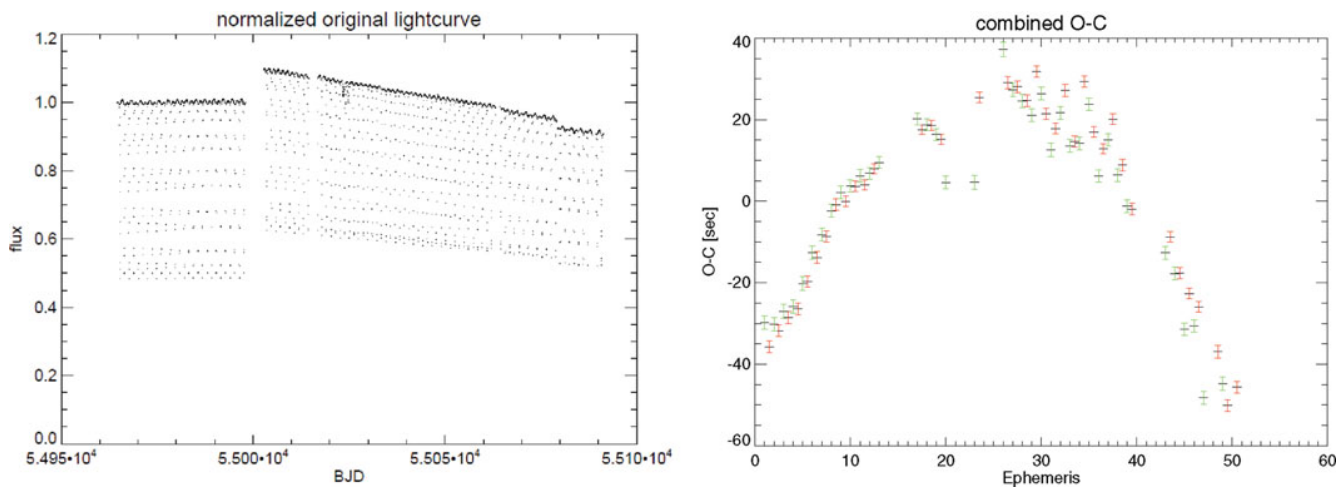

Figure 2. Normalized light curve and O-C diagram of KID6543674.

\section{Conclusions}

The presence of a third body in the system is one of the possible explanations for TVs in eclipsing systems (light time effect). On the other hand, they can also result from pulsations of the components, magnetic activity, precession of the orbits and mass transfer between the components. The corrected light curves of the Kepler EBs and the new $\mathrm{O}-\mathrm{C}$ diagrams will be presented soon, in order to confirm that the high photometric precision and duty cycle of space missions allow us to obtain the best results in the detection of low mass substellar objects via ETVs (Schwarz et al. 2011).

\section{References}

Prša, A., Batalha, N., Slawson, R. W. et al., 2011, AJ, 141, 83

Schwarz, R., Haghighipour, N., Eggl, S., Pilat-Lohinger, E., \& Funk, B. 2011, MNRAS, 414, 2763

Slawson, R. W., Prša, A., Welsh, W. F., et al., 2011, AJ, 142, 160 